GORANA BIKIĆ-CARIĆ

Université de Zagreb

\title{
L'ASPECT VERBAL EN FRANÇAIS ET EN CROATE
}

\begin{abstract}
Bikić-Carić Gorana, L'aspect verbal en français et en croate [The verbal aspect in French and Croatian]. Studia Romanica Posnaniensia, Adam Mickiewicz University Press, Poznań, vol. XXXI: 2004, pp. 167-174. ISBN 83-232-1353-4, ISSN 0137-2475.

The fact that French and Croatian belong to different linguistic families implies some specific problems for non-native speakers, one of which is the notion of aspect. In this paper we would like to compare how the idea of aspect is represented in Croatian and in French. We will also show how aspect in Croatian can be an equivalent of the subjonctif in French.
\end{abstract}

\section{INTRODUCTION}

Le croate étant une langue slave, son système verbal repose, en grande mesure, sur la notion d'aspect. Nous oserions même dire que c'est la notion principale, beaucoup plus importante que l'antériorité ou la postériorité. Par contre, le français, comme une langue romane, suit un système essentiellement différent.

Dans le système verbal français (et nous allons nous limiter à la langue quotidienne, donc non soutenue), on distingue, pour exprimer le passé, l'imparfait, le passé composé, et le plus-que-parfait. Pour exprimer le présent - une seule forme verbale, le présent, et pour le futur - le futur simple et le futur antérieur (nous ne tenons pas compte ici des périphrases verbales). Quels sont les critères pour distinguer l'emploi de ces formes verbales? Pour le futur et pour le plus-que-parfait, ce sont l'antériorité et, dans une certaine mesure, l'accomplissement du fait. Le passé composé et l'imparfait, quant à eux, s'opposent principalement sur le critère de l'accomplissement du fait et sur l'opposition action/description.

Par contre, le croate repose sur un système différent. Dans la langue quotidienne, nous pourrions presque limiter l'expression du passé à une seule forme verbale: le perfekt (le plus-que-parfait est très rare dans la langue de tous les jours, et l'aorist et l'imperfekt sont très littéraires, beaucoup plus que le passé simple en français). Pour exprimer le présent, une seule forme verbale (le prezent), et pour 
exprimer le futur, deux - le futur simple (futur prvi) et le futur antérieur (futur drugi). A la différence du français, dans la langue croate l'antériorité joue un rôle presque négligeable (c'est pourquoi le plus-que-parfait est très rare dans la langue quotidienne, et le futur antérieur plutôt rare aussi). Dans une certaine mesure, l'accomplissement du fait et l'opposition action/description se trouvent en croate aussi, mais ces notions ne se correspondent pas toujours dans les deux langues. Par contre, dans le croate ce sont les aspects qui jouent le rôle primordial dans le système verbal. Presque tous les verbes ont au moins une opposition: l'aspect perfectif/imperfectif, et cela à partir de l'infinitif déjà (donc, ceux qui veulent aprendre le croate doivent apprendre au moins deux infinitifs pour presque tous les verbes). C'est pourquoi il suffit d'utiliser un seul temps, le perfekt, pour exprimer le passé (et c'est pourquoi l'aorist et l'imperfekt ont disparu de l'usage quotidien, et même, dans une grande mesure, de la langue écrite contemporaine). Nous pourrions dire que le problème pour les apprenants réside justement dans le fait que les systèmes verbaux des deux langues ont des points communs, mais en même temps représentent de grandes différences.

\section{LA NOTION D'ASPECT EN FRANÇAIS}

La conception de la notion d'aspect en français varie selon les auteurs. Comme nous sommes limités par l'espace, nous n'allons mentionner que Grevisse et Goosse (1995: 243). D'après eux, les principaux aspects en français sont: instantané (La bombe éclate), duratif (J'écrivais quand elle est entrée), inchoatif (Elle s'endort), itératif (Il buvote son vin), accompli ou achevé (J'ai écrit ma lettre), récent (Elle vient de mourir), imminent (Je vais partir).

Comme nous pouvons voir dans ces exemples, les aspects ne se distinguent pas par une forme particulière du verbe (excepté dans l'opposition s'endormir/dormir ou buvoter/boire - des cas pareils sont plutôt une exception en français), mais par la forme verbale ou le «temps» (surtout l'opposition imparfait/passé composé) ou, tout simplement, par le sens du verbe (La bombe éclate).

Pour Damić Kruk (1992: 45), le systeme verbal français repose sur un système temporel qui connaît seulement au passé l'opposition des aspects perfectif et imperfectif, celle-ci étant réalisée par l'opposition passé simple (passé composé)/imparfait. Cette opposition, à elle seule, répond aux critères établis: catégorie formelle qui exprime la manière dont se déroule le procès, la perfectivité des temps composés étant impliquée par l'antériorité.

Même dans l'opposition imparfait/passé composé, il ne faut pas oublier que les choses ne sont pas toujours simples. En effet, l'imparfait peut avoir des emplois qui sont tout à fait opposés à son idée principale d'imperfectivité. Un exemple: En juin, brusquement, son frère mourait. 


\section{LA NOTION D'ASPECT EN CROATE}

Damić Bohač (1997: 11) affirme qu'on peut parler de système aspectuel à condition que l'aspect assume une fonction commune nécessaire à tout l'ensemble et que le locuteur soit constamment obligé de choisir un aspect. En d'autres termes chaque procès verbal doit être marqué aspectuellement. Le croate en est un bon exemple.

En croate, les verbes expriment l'aspect perfectif et imperfectif dans toutes leurs formes verbales (ou «temps»), et c'est la différence essentielle par rapport au français. En effet, ce n'est pas le temps ou la forme verbale qui est le porteur de l'aspect, mais le verbe lui-même. En plus, les verbes, à l'aide de la préfixation ou de l'infixation, expriment aussi de différentes manières dont l'action verbale se déroule. D'apres Barić et al (Barić et al, 1979: 146), les verbes peuvent être:

a) pantifs - expriment une action graduelle, dont le résultat est visible dans chaque phase (gradi kuću-sagradio je trećinu, polovinu, svu kuću/il construit une maison - il en a construit un tiers, la moitié, toute la maison);

totifs - expriment une action qui couvre la totalité de l'objet da manière que les résultats de chaque phase ne sont pas visibles (kuhao je mlijeko - skuhao je mlijeko / il faisait bouillir le lait - il a fait bouillir le lait);

inchoatifs - expriment le début d'une action (poći, polaziti / partir);

finitifs - expriment la fin d'une action (dopuniti, dopunjavati / compléter);

b) comparatifs, qui, à leur tour, se divisent en:

diminutifs - expriment une action d'intensité inférieure à celle d'une action habituelle (grickati, gricnuti/grignoter);

augmentatifs - expriment une action d'intensité supérieure à celle d'une action habituelle (prejesti se, prejedati se / trop manger);

majoratifs - expriment une action qui surpasse une autre action (nadvikati, nadvikivati / crier plus fort que quelqu'un d'autre);

c) intensifs --expriment une action de grande intensité qui atteint son sommet (razveseliti se, razveseljavati se / sentir une grande joie);

satifs - expriment une action qui suffit au sujet (najesti se, najedati se / manger à volonté);

d) duratifs - expriment une action qui peut durer sans arrêt (donc, ces verbes sont tous imperfectifs: boriti se /lutter, cvjetati /fleurir, živjeti / vivre);

itératifs - expriment une action qui se répète, c'est-à-dire qui s'arrête et puis continue (odlijetati, poodlijetati / s'envoler un à un);

momentanés - expriment une action qui se déroule dans un laps de temps très court (donc, ces verbes sont tous perfectifs: jurnuti / se précipiter).

Comme nous venons de voir, dans la majorité des paires de verbes perfectifs/imperfectifs, il existe aussi une autre opposition outre que la perfectivité. Vu ce chevauchement entre le sens sémantique et grammatical, certains grammairiens parlent de sous-aspects des aspects perfectif et imperfectif. 


\section{L'ASPECT ET LES TEMPS VERBAUX EN CROATE}

Au début de cet article, nous avons souligné qu'en croate tous les temps ou formes verbales de la langue quotidienne peuvent être perfectifs ou imperfectifs.

Le "vrai» présent, exprimant nécessairement une action en train de se dérouler, n'admet que l'aspect imperfectif. Mais, dans ses autres emplois, le présent admet aussi l'aspect perfectif. Par exemple, dans des propositions subordonnées:

a) de temps:

Kada završišs, moći ćeš otići. (Quand tu auras terminé, tu pourras partir.)

b) de condition:

Ako završiš, možeš otići. (Si tu termines, tu peux partir.)

On peut employer le présent d'un verbe perfectif même dans une phrase principale ou indépendante, pour exprimer une habitude ou le présent historique. Par exemple:

Svaki put kad ga pogledam, zadrhtim. (Chaque fois que je le regarde, je tressaillis.)

Pogledam ga - on se ne mixe. (Je le regarde - il ne bouge pas.)

Quant aux temps du passé, comme nous avons déjà remarqué, le plus souvent on peut remarquer la correspondance passé composé (ou passé simple)/aspect perfectif, imparfait/aspect imperfectif. Mais, et voici un problème pour les apprenants croatophones, ce n'est pas toujours le cas. Par exemple, dans la phrase: Il a fumé pendant quinze ans, le locuteur francophone emploie le passé composé, parce que pour lui, ce qui compte, c'est la durée limitée du fait. Par contre, pour un croatophone, il s'agit de la durée tout court, si ce n'est pas tout simplement une description de quelqu'un, de sa vie (même de son état à l'époque), et non d'une action délimitée ou terminée. C'est pourquoi il est impossible d'employer l'aspect perfectif dans la traduction de cette phrase en croate. Par contre, il faut dire: Pušio je petnaest godina.

Quant au futur, nous nous bornerons, en croate, au futur simple des verbes perfectifs et imperfectifs. Le futur antérieur (futur drugi) existe aussi, mais son emploi est en général limité aux propositions subordonnées de temps (quand...) ou de condition (si...). En français, le futur drugi se traduit par le futur antérieur après "quand» et du présent après «si». Par contre, le futur simple en croute est bien capable d'exprimer les nuances des deux futurs en français. Par exemple:

Sutra éu pisati pismo. - Demain j'écrirai une lettre.

Sutra ću napisati pismo. - Demain j'aurai écrit la lettre.

Brod će dugo nestajati. - Le bateau mettra du temps à disparaitre.

Brod će nestati za sat vremena. - Le bateau aura disparu dans une heure.

Mais, ce n'est pas toujours le cas, c'est-à-dire, le futur antérieur ne correspond pas systématiquement au futur d'un verbe perfectif, ni le futur simple au futur d'un 
verbe imperfectif. Loin s'en faut. Par exemple: Je vous paierai la semaine prochaine ne peut être traduit que par la forme perfective, puisqu'il s'agit d'un fait qui sera accompli et momentané (Platit ću vam sljedećeg tjedna.)

\section{LE MODE EN FRANÇAIS DEVIENT L'ASPECT EN CROATE ET VICE-VERSA}

Dans certains cas, dans des propositions subordonnées, l'aspect perfectif du verbe au présent correspond au subjonctif en français. Par exemple:

a) conjonctive essentielle:

Važno je da krenemo rano. (Il importe que nous partions tôt.)

Spriječite da ona ne izađe. (Empêchez qu'elle ne sorte.)

Moguce je da vlak zakasni. (Il est possible que le train soit en retard.)

MAIS: Moguće je da vlak kasni. - l'aspect imperfectif, par contre, exprime un fait réel, le train est peut-être déjà en retard, même si nous ne le savons pas

Želim da dođe. (Je veux qu'il vienne.)

b) adverbiale de temps:

Ostat ću ovdje dok se ne vratite. (Je resterai ici jusqu'à ce que vous ne reveniez.)

c) adverbiale de manière:

Razdijelio je kolax tako da svi budu zadovoljni. (II a partagé le gâteau de manière que tout le monde soit satisfait.)

MAIS: Razdijelio je kolaè tako da su svi zadovoljni. (Il a partagé le gâteau de manière que tout le monde est satisfait.)

d) adverbiale de but:

Dala mu je bombon da bude miran. (Elle lui a donné un bonbon pour qu'il se tienne tranquille.)

e) adverbiale de condition:

Oprostit ćemo ti ako se ispričă. (On te pardonnera, pourvu que tu fasses des excuses.)

Nous pourrions conclure que l'aspect perfectif du présent généralement n'exprime pas une réalité, mais une action envisagée dans l'esprit du locuteur (comme une condition, un but, une supposition etc.). C'est pourquoi nous avons pensé à le rapprocher du subjonctif en français (et en général dans la plupart des langues romanes). 


\section{COMPARAISON DE TEXTES TRADUITS}

Pour illustrer nos propos, nous avons décidé de comparer un texte en français avec sa traduction en croate, et un texte en croate avec sa traduction en français. Dans les chapitres suivants, nous ne marquons pas les cas qui suivent la règle, c'està-dire ceux où le passé composé/le passé simple en français correspondent au perfekt des verbes perfectifs en croate, et où l'imparfait en français correspond au perfekt des verbes imperfectifs en croate. Nous avons marqué tous les autres cas: les exceptions à cette règle, ainsi que les occurences où l'aspect perfectif en croate correspond au subjonctif en français. De même, nous marquons les occurences où la notion d'aspect se perd en français, ou s'exprime en employant un autre verbe, ou bien les occurences où le plus-que-parfait se perd en croate.

\section{Ranko Marinković: Zagrljaj (L'Etreinte)}

Drži se tvoja Rečenica kao klisurina. Gradio si je cvrstim, zbijenim rukopisom, (...) pa se iz svoje ogromne nadmoćnosti samilosno smješkaš bijednom mravu što hoće da ti ukrade tocku... (Marinković, 1966: 178)

Ta phrase se maintient comme un roc. Tu l'as bâtie avec une écriture robuste, serrée, (...) alors de ta colossale supériorité tu souris avec compassion à la fourmi misérable qui cherche à t'enlever le point... (Marinković, 1994: 10)

Gradio si je - Tu l'as bâtie (contrairement à ce qu'on pourrait supposer, l'aspect imperfectif est traduit par le passé composé); smješkaš se - tu souris (l'aspect itératif se perd en français)

Nezanimljivo, svakidasnje! Pogled ti je pun prezriva gađenja. Kako ces na tome izgraditi pricèu? Ni pas da zalaje na ovu zacaranu, tvrdoglavu nepomix̌nost! Da barem netko zavikne «vatra!», da razbije staklo! Desilo bi se možda nešto dramatično, živo, vrijedno pažnje. Vrijedno da se odšarafi "parker". (Marinković, 1966:179)

Pas intéressant, quotidien! Ton regard est rempli de dégoût dédaigneux. Avec ça, comment vas-tu construire une histoire? Même pas un chien qui aboie sur cette immobilité envoûtée, obstinée! Si seulement quelqu'un criait: "au feu'» et cassait une vitre! Il arriverait peut-être quelque chose de dramatique, de vivant, digne d'attention. Digne qu'on dévisse son "parker» (Marinković, 1994: 12).

izgraditi - construire (l'aspect perfectif en croate se perd dans l'infinitif présent en français); zalaje - aboie, zavikne - criait, razbije - cassait, Desilo bi se - arriverait (l'aspect perfectif des verbes croates se perd en français); odšarafi dévisse (l'aspect perfectif se traduit par le subjonctif)

Kiša je dobra, ona je i sama podsmješljiva. (...) A onda ce odjedanput pljusnuti, i bit ćemo mokri i Zivahni kao vrapci (Marinkovič, 1966: 198). 
La pluie est bonne, elle est même drôle. (...) Et puis d'un coup elle va se mettre à nous arroser, et nous serons mouillés et fringants, comme des moineaux. (Marinković, 1994: 39)

...će pljusnuti - va se mettre à nous arroser (l'aspect perfectif au futur est traduit par une périphrase verbale)

\section{Marguerite Duras: Un barrage contre le Pacifique (Brana na Pacifiku)}

La mère changea d'avis: elle décida qu'ils ne devaient plus rester seuls à l'intérieur du bungalow, même avec la porte ouverte. Sans doute trouvait-elle que ce n'était plus suffisant pour exaspérer l'impatience de M. Jo. Du moment que celui-ci attendait toujours on ne savait quoi, disait-elle, alors qu'elle le savait fort bien, pour faire sa demande en mariage, ce n'était plus suffisant.

C'était donc sur les talus qui bordaient le rac, à l'ombre du pont, que Suzanne recevait $M$. Jo. Tous attendaient qu'il se décide. La mère lui avait parlé et lui avait donné huit jours pour le faire. $M$. Jo avait accepté le délai. Il avoua à la mère que son père avait pour lui d'autres projets et que bien qu'il y eût peu de jeunes filles, dans cette colonie, de fortune digne de la sienne, il y en avait quand même suffisamment pour qu'il lui soit très difficile de faire fléchir son père. Il lui promit cependant d'employer toutes ses forces pour y arriver (Duras, 1950: 113).

Majka se predomislila: odluèila je da ne smiju više ostajati sami u bungalovu, pa ni uz otvorena vrata. Vjerojatno je smatrala da to više nije dovoljno kako bi se raspirilo nestrpljenje gosp. Joa. S obzirom da je on joś uvijek cekao tko zna što, govorila je ona, iako je vrlo dobro znala što, to više nije bilo dovoljno da on zaprosi njezinu ruku.

Sada ga je Suzana doèekivala na padinama riječnoga rukavca, u sjeni mosta. Svi su čekali da se on odtuci. Majka je s njim razgovarala i dala mu je rok od tjedan dana da to ucini. Gosp. Jo je prihvatio taj rok. Priznao je majci da otac za njega ima druge planove, pa iako u toj koloniji ima malo djevojaka čije je bogatstvo dostojno njihova, ipak ih ima dovoljno, $i$ stoga mu je neobicno teško nagovoriti oca da popusti. Ipak joj je obećao da ce upotrijebiti sve svoje snage da to postigne (Duras, 2002: 86).

rester - ostajati (le verbe dans la traduction croate exprime l'aspect imperfectif, qui n'est pas marqué dans l'original français); exaspérer - bi se raspirilo, faire sa demande en mariage - zaprosi njezinu ruku (le verbe en croate exprime l'aspect perfectif, qui n'est pas marqué dans l'original français); se décide - se odluči (le subjonctif en français est traduit par l'aspect perfectif en croate); avait parlé - je razgovarala, avait donné - dala je, avait accepté - je prihvatio (le plus-que-parfait en français se perd en croate); faire - učini, faire fléchir son père - nagovoriti oca da popusti, employer - upotrijebiti, arriver - postigne (les infinitifs en français n'expriment pas l'aspect, qui est perfectif dans la traduction croate)

- Ça serait complètement inutile d'aller à Kam, reprit-elle, je vais les garder pour moi (Duras, 1950: 253).

- Bilo bi posve uzalud ići u Kam, nastavi ona, sačuvat ću ih za sebe (Duras, 2002: 200). 
vais les garder - sacuvat ću ih (le verbe en croate exprime l'aspect perfectif, qui n'est pas marqué en français; par contre, la valeur du futur proche se perd en croate).

\section{CONCLUSION}

Dans cette intervention nous avons essayé de comparer une notion qui est essentielle en croate et marginale en français. Il est évident que le sujet est très complexe et mérite une attention particulière, non seulement parce qu'il s'agit d'un problème non négligeable pour les apprenants des deux langues, mais aussi parce que, si on s'y penche un peu plus sérieusement, on peut découvrir des correspondances inattendues. Les langues expriment souvent, d'une façon ou d'une autre, une certaine sélection de la part de leurs locuteurs face à la réalité plus ou moins commune à tous. Nous croyons pouvoir dire que c'est justement ce fait qui rend l'étude des langues tellement passionnante.

\section{BIBLIOGRAPHIE}

B arić, E. et al (1979), Priruèna gramatikn hrvatskoga književnog jezika. Zagreb: Skolska knjiga.

Damić Bohač, D. (1997), Sur la définition du verbe et des catégories grammaticales. Strani jezici, $\mathrm{XXV} / 1,9-13$.

Damić Kruk, D. (1991 - 1992), L'aspect verbal en français. SRAZ XXXVI-XXXVII, 37-45.

Duras, M. (1950), Un barrage contre le Pncifique. Paris: Editions Gallimard.

Duras, M. (1950), Brana na Pacifiku, trad. Ingrid Śafranek. Zagreb: Vuković \& Runjić, 2002.

Grevisse, M. et Goosse, A. (1995), Nouvelle grammaire française. Louvain-la-Neuve: DeBoeck \& Duculot.

Marinković, R. (1966), Novele. Zagreb: Skolska knjiga.

Marinković, R, (1966), L'Etreinte. trad. Sineva Béné. Zagreb: Društvo hrvatskih književnika, 1994. 